\title{
Effect of Atmospheric Plasma Paint Removal on the Fatigue Performance of 2024-T3 Aluminium Alloy Sheet
}

\author{
Marko Yanishevsky, Ali Merati, Yan Bombardier \\ National Research Council Canada, NRC Aerospace, Ottawa, Canada \\ Email: ali.merati@nrc-cnrc.gc.ca
}

How to cite this paper: Yanishevsky, M., Merati, A. and Bombardier, Y. (2018) Effect of Atmospheric Plasma Paint Removal on the Fatigue Performance of 2024-T3 Aluminium Alloy Sheet. Journal of Minerals and Materials Characterization and Engineering, 6, 15-24.

https://doi.org/10.4236/jmmce.2018.61002

Received: November 17, 2017

Accepted: December 25, 2017

Published: December 28, 2017

Copyright $\odot 2018$ by authors and Scientific Research Publishing Inc. This work is licensed under the Creative Commons Attribution International License (CC BY 4.0).

http://creativecommons.org/licenses/by/4.0/

(c) (i) Open Access

\begin{abstract}
This article documents fatigue testing that was conducted using as-painted (baseline) and Atmospheric Plasma de-painted specimens made of 0.063 inch thick 2024-T3 aluminium alloy sheet. The intent of the test program was to determine whether AP de-painting would alter the fatigue properties of this aluminium substrate. AP de-painting process parameters were selected based on previous work that would remove the topcoat, while leaving most of the primer intact. This process was repeated five times to simulate service experience, where aircraft typically undergo five paint/de-paint cycles in their lifetime. As-painted (baseline) and five times de-painted specimens were fatigue tested under constant amplitude conditions, at two load ratios and several maximum stress levels. Ten samples per condition were used to establish statistical behaviour and repeatability. The test results and statistical analysis demonstrated that the selected AP process parameters did not have a detrimental effect on the fatigue performance of 2024-T3 aluminium alloy sheet.
\end{abstract}

\section{Keywords}

Atmospheric Plasma, De-Painting, Fatigue Performance, Five Paint/De-Paint Cycles, 2024-T3 Aluminium Sheet

\section{Introduction}

Aircrafts are typically coated with highly durable paint schemes to provide reliable corrosion protection, and to enhance their appearance. Unfortunately, over time these coatings deteriorate and become damaged due to environmental exposure and service related wear, which then require restoration. As well, critical structures that require inspection for damage, in most cases require direct access 
to the substrate material surface with the paint scheme fully removed. As such, aircraft will undergo numerous removal and re-application cycles during their service lifetime to restore appearance, corrosion protection, or to enable inspection for fatigue cracks and corrosion damages. Current approved paint removal processes include chemical and abrasive media blasting. These processes yield high amounts of volatile organic compounds and generate large quantities of waste, which require proper disposal/treatment. Hand sanding is also an approved process for removing paint from delicate composite structures; however, the process is labour-intensive and can lead to worker muscular-skeletal disorders.

Atmospheric Plasma (AP) de-painting is a novel environmentally friendly, alternative paint removal method based on using a relatively cold air plasma. Plasma is considered the fourth state of matter and air plasma contains ionized nitrogen and oxygen and other chemically reactive species, such as monatomic oxygen, capable of effectively oxidizing organics in paint schemes and converting them to water vapour and carbon dioxide. The AP process uses dry compressed air and electricity to create these chemically reactive species and requires a suction filtration system to filter and collect the resultant waste debris consisting of inorganic powders contained in the original paint. This waste debris is only a small fraction of the original paint scheme volume.

Typically an aircraft will undergo at least five paint/de-paint cycles in its lifetime. As part of understanding whether Atmospheric Plasma (AP) paint removal affects the material properties of aluminium alloys used in aerospace applications, NRC chose to undertake a fatigue study in accordance with requirements described in the Society for Automotive Engineers (SAE) MA4872A, Paint Stripping of Commercial Aircraft-Evaluation of Materials and Processes [1]. In this document, a minimum of five paint/de-paint cycles are required for the comparison between baseline (painted) and de-painted material.

Since there was no access to repainting facilities for its evaluation, a slightly different approach to the above was undertaken. Because the AP setup cycle can be adjusted to remove the topcoat only and retain most of the primer, the cycle parameters for such a result were repeated five times in succession. This was considered to be a conservative approach, as the substrate was not re-protected with primer touch-up and full topcoat, and any negative effects of AP processing would be amplified on the substrate, one of the potentially detrimental effects being heat exposure. To address this worse-case scenario, NRC performed the AP paint removal process five times in succession without allowing the test stage and substrate to cool properly between cycles.

\section{Experimental Approach and Material}

The Atmospheric Plasma (AP) System manufactured by Atmospheric Plasma Solutions Inc. (Cary, NC, USA) is shown in Figure 1 and Figure 2. The system consists of the atmospheric plasma generating system, a 3-axis robotic stage 


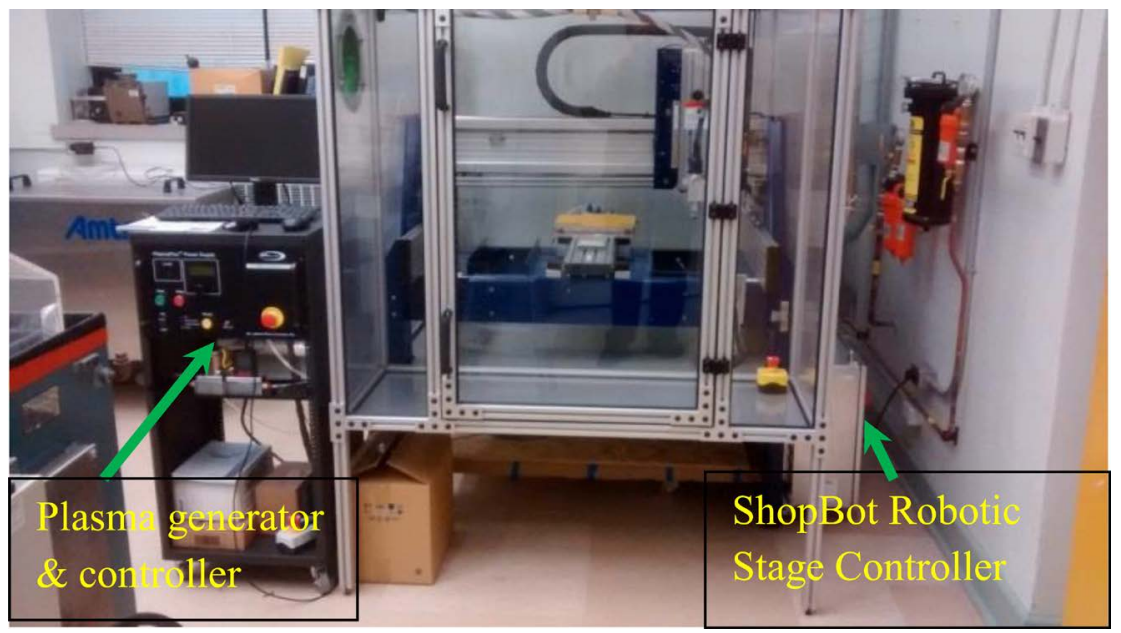

Figure 1. Front view of Atmospheric Plasma System.

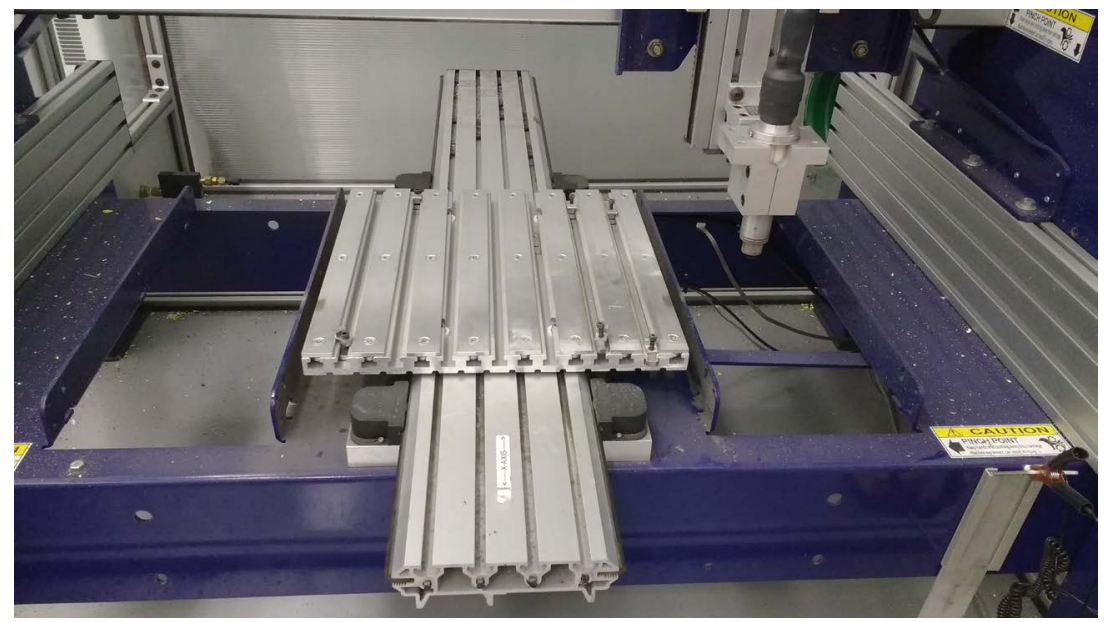

Figure 2. Large slotted table with mechanical screw clamps for securing specimens.

manufactured by ShopBot Tools Inc. (Durham, North Carolina, USA) that is used to provide movement of the plasma pen, and an enclosure used to isolate the working area. The controllers of both systems are shown in Figure 1.

A single large slotted table, shown in Figure 2, was used to secure the specimens using mechanical screw clamps.

The 48 " wide by 72 " long aluminium alloy sheets used for this project were manufactured by Kaiser Aluminium and supplied by New Source Corporation. The supplied materials were cleaned, primed and painted using qualified staff at the Quality Engineering Test Establishment (QETE) of the Canadian Department of National Defence (DND). MIL-PRF-23377 Type I Class N primer and MIL-PRF-85285 Type I Class H topcoat were used.

The 2024-T3 substrate with a 0.063 inch thickness was selected to study the effect of five de-painting cycles using Atmospheric Plasma on its fatigue properties. Panels with matte (flat) black and glossy (shiny) black paint were selected for the study, as shown in Figure 3. Portions of the panels were identified for baseline and AP treatment purposes. 


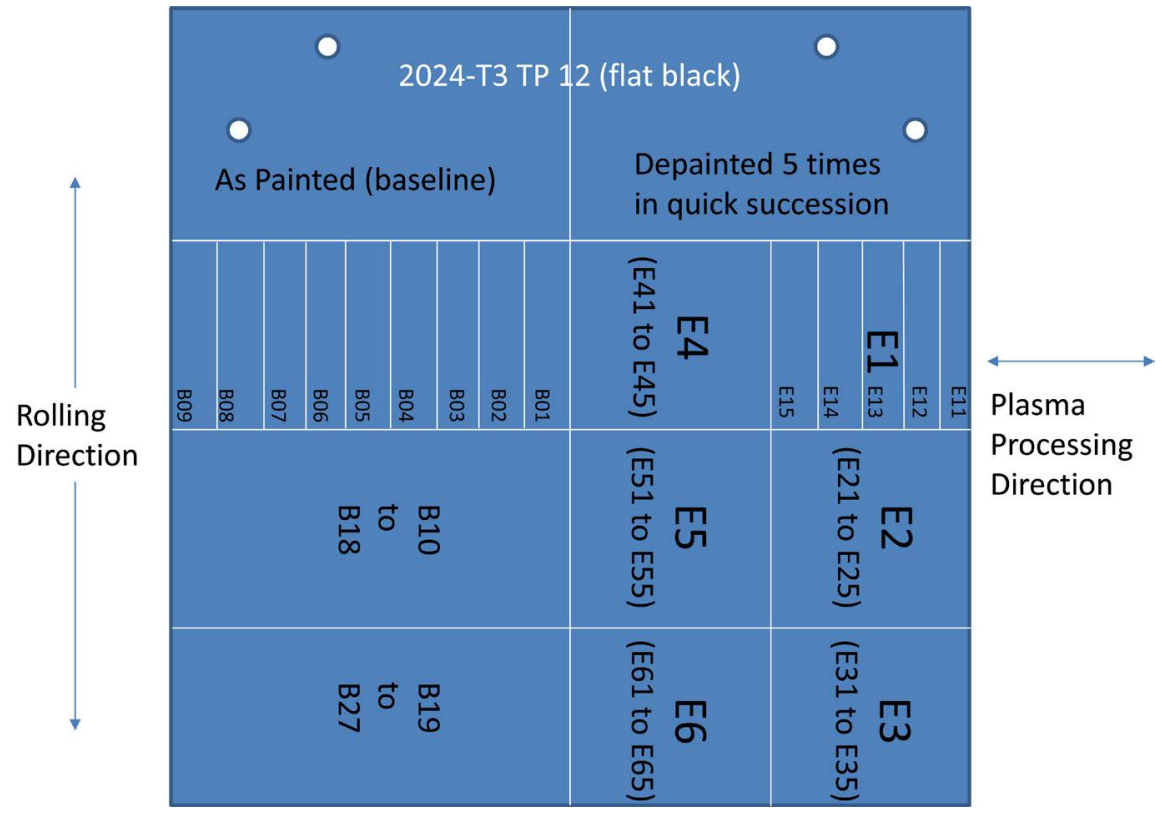

Figure 3. Specimen designations for matte (flat) black painted panels. Baseline specimens were removed from the left side. The right side was cut into six 6 inch $\times 6$ inch sections, which were AP de-painted five times, and then cut into de-painted specimens.

Table 1. AP De-painting processing parameters.

\begin{tabular}{cc}
\hline Height above substrate, (inch) & 0.25 \\
Speed, (inches/second) & 10 \\
Number of passes & 1 \\
Increment, (inch) & 0.02 \\
\hline
\end{tabular}

\subsection{Five Cycles De-Painting}

The AP process parameters selected to adequately remove the top coat layer, while retaining the primer after one de-painting cycle, were predetermined from previous work [2]-[9]. The parameters used are listed in Table 1.

The 6 inch by 6 inch cut sheets that were to be de-painted, were mounted two aside on the slotted table mounted on the ShopBot stage. The ShopBot robot was programmed to move the plasma pen 24 inches from side to side. The 12 inches of painted specimens were mounted within this 24 inches. Since the effective width of each pass is approximately $0.1 \mathrm{inch}$, these processing parameters effectively had the plasma plume go over the same area of the panels approximately five times per de-painting cycle. At the fastest rate available for the ShopBot robot, 10 inches/second, it took 15 minutes to de-paint a 5 inch length. The plasma has a localized temperature of approximately 230 degrees Celsius. As all five de-painting cycles were conducted in quick succession, the panels effectively had the plasma plume pass over the same areas 25 times, for a total exposure time of 1 hour 15 minutes for all five cycles, without an opportunity for both the test specimens and the test stage to cool between cycles. Since all five paint de-painting cycles were performed in succession without repainting, not only was the top- 
coat removed, but ultimately the underlying primer was removed as well. This would not have occurred had an intermediate primer touch-up and repaint step between de-painting cycles been included. Since the substrate was not allowed to cool and was not reprotected by additional primer plus topcoat between paint cycles, this type of exposure to the plasma plume was considered to be a worst case representation of the paint/de-paint scenario.

\subsection{Fatigue Specimens and Test Matrix}

The fatigue specimen design was based on the American Society for Testing and Materials ASTM E466 Standard Practice for Conducting Force Controlled Constant Amplitude Axial Fatigue Tests of Metallic Materials [10]. The specimen dimensions were: 6 inches in overall length, 1 inch width in the grip areas, a 1 inch gauge length, and a gauge width of 0.4 inches. Baseline and de-painted configuration specimens were machined and marked as per the schematics shown in Figure 3.

Based on available data in Metallic Materials Properties Development and Standardization (MMPDS) [11], stress levels were selected to achieve approximately a range of fatigue lives between 50,000 and 1,000,000 cycles. For statistical significance 10 specimens for each stress level in both baseline and de-painted configurations were tested, primarily focusing on a load ratio (minimum load/ maximum load), $R=0.1$. A limited number of stress levels were also tested at a higher load ratio, $R=0.5$. Testing was conducted at frequencies of 20 and $25 \mathrm{~Hz}$.

The anticipated cycles to failure for the load levels and $R$ ratios chosen were determined using the equivalent stress equation best fit provided on page 3-166 of MMPDS [11]. The maximum stress level of 56, 52.8, 49.6 and $46.40 \mathrm{ksi}$ for the stress ratio of $R=0.1$, and two maximum stress level of 62 and 56 ksi for the stress ratio of $R=0.5$ were selected. The full detail of test results is provided in reference [13].

\section{Results and Discussion}

\subsection{Statistical Analysis of Fatigue Data}

This section addresses the statistical analyses of the fatigue data obtained for the baseline and AP de-painted specimens based on ASTM E739 standard [12]. The graphs in Figure 4 are comparing the Stress-Life curves obtained for $R=0.1$ and $R=0.5$. The confidence curves, shown using the dashed lines, were calculated using a 95\% confidence level according to ASTM E739. As shown, the confidence level did not encompass the high stress fatigue results at $R=0.1$. Consequently, lower and upper bounds should be used with care for the data sets and other approaches for describing the lower and upper bounds should be evaluated. Overall, the comparison of the average Stress-Life curves presented in Figure 4 suggests that the plasma process did not affect the fatigue life of the tested specimens as the curves obtained from the plasma de-painted specimen results fall close to the baseline curves and within their calculated confidence bands. 


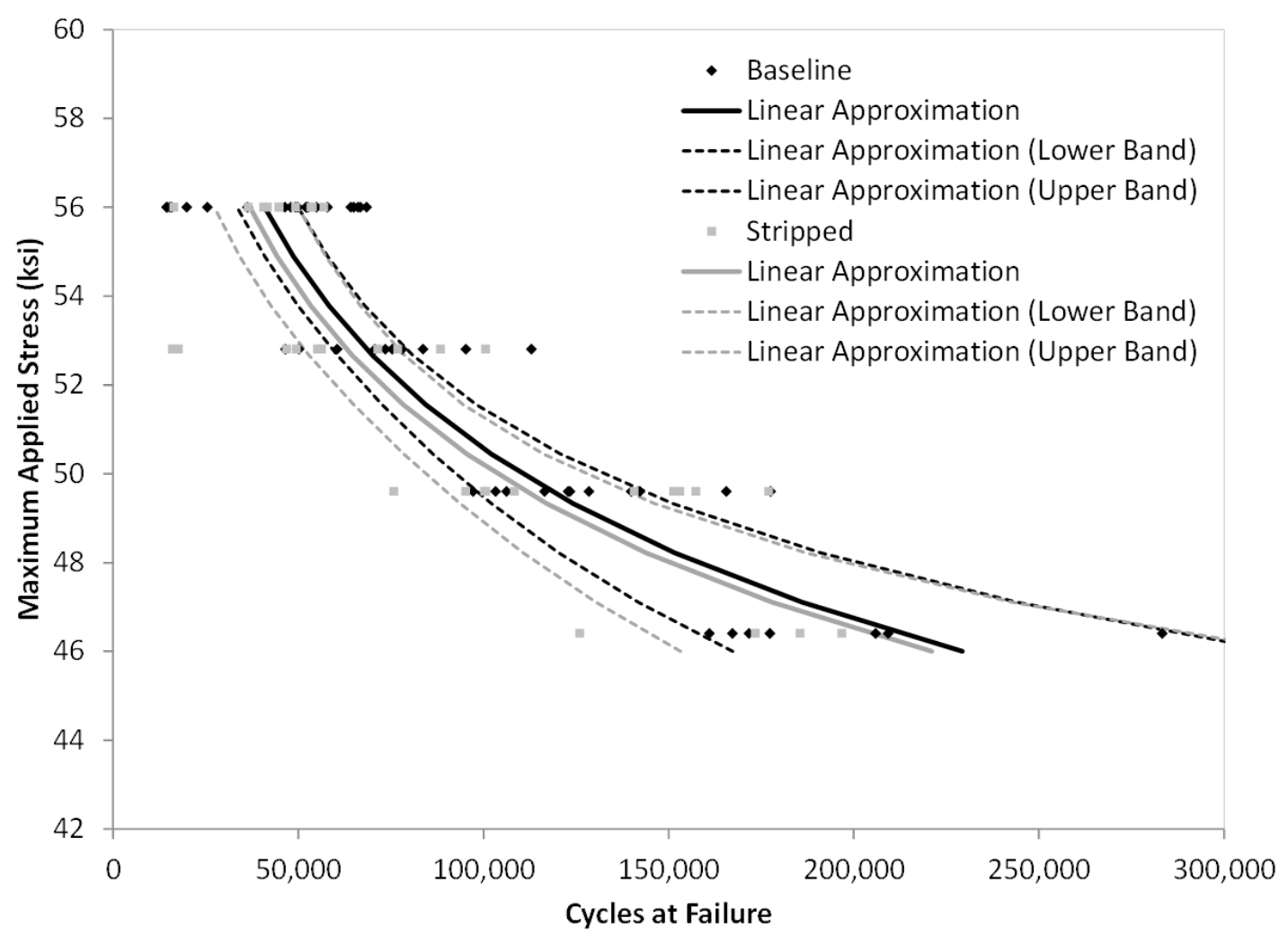

(a)

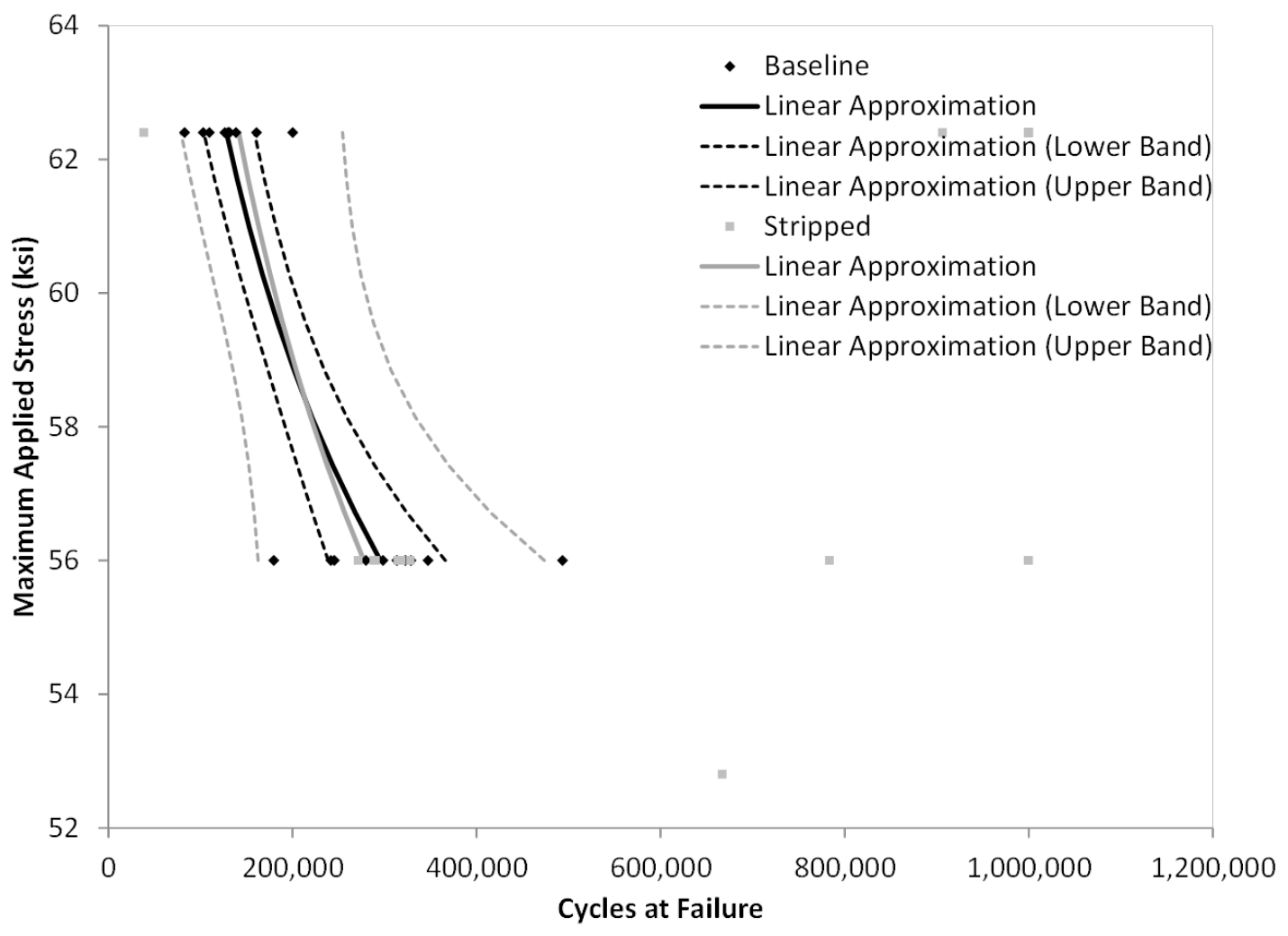

(b)

Figure 4. Experimental data and fitted relationship between the fatigue life and the maximum applied stress for two tested stress ratios. (a) Stress ratio of 0.1 ; (b) Stress ratio of 0.5 . 


\subsection{Fractography}

The objective of fractography was to determine whether paint removal using Atmospheric Plasma would change or shift the fatigue crack nucleation sites to the de-painted, as-painted surfaces of the fatigue specimens. The fracture surfaces of all the test specimens were examined using an optical microscope to determine fatigue nucleation sites and features. Some fracture surfaces were also further examined using a scanning electron microscope (SEM). Fractography of all failed specimens investigated are presented in reference [13].

Examples of a typical fracture surface for the de-painted and as-painted (not de-painted) specimens are shown in Figure 5 and Figure 6. The nucleation sites

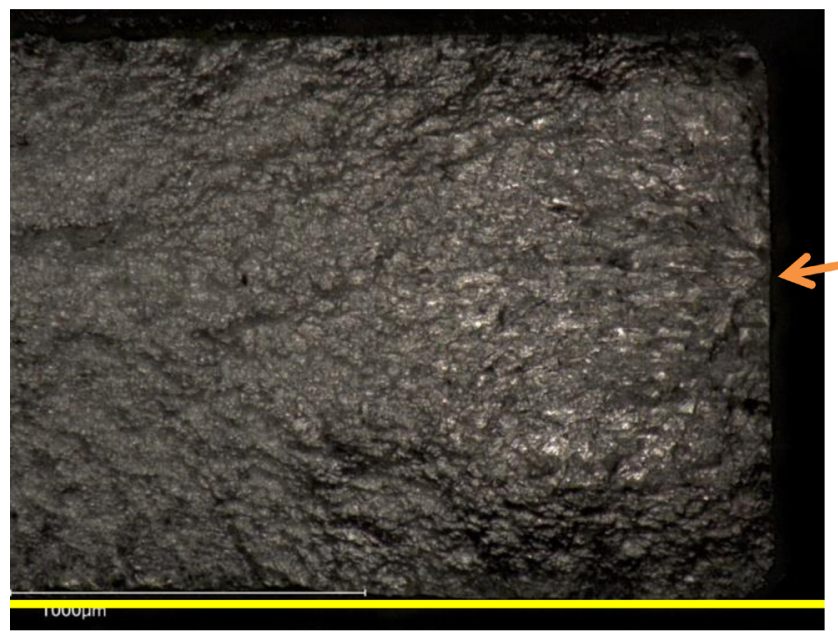

(a)

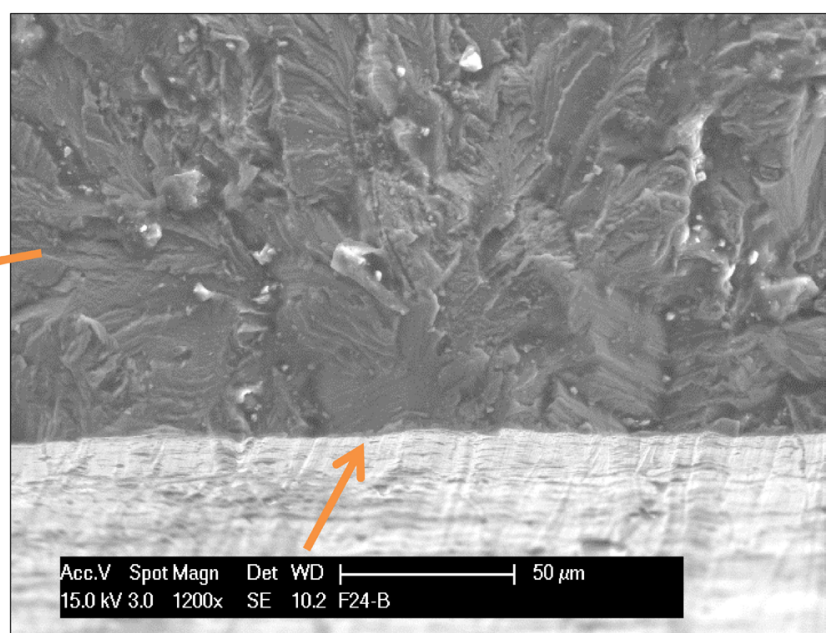

(b)

Figure 5. Fracture surface of the de-painted fatigue specimen F24. (a) De-painted face on bottom (designated with the yellow line). The arrow is pointing to the nucleation site at the edge (thickness of specimen); (b) Higher magnification of nucleation site under SEM, indicating a potential particle as source of fatigue crack nucleation (orange arrow).

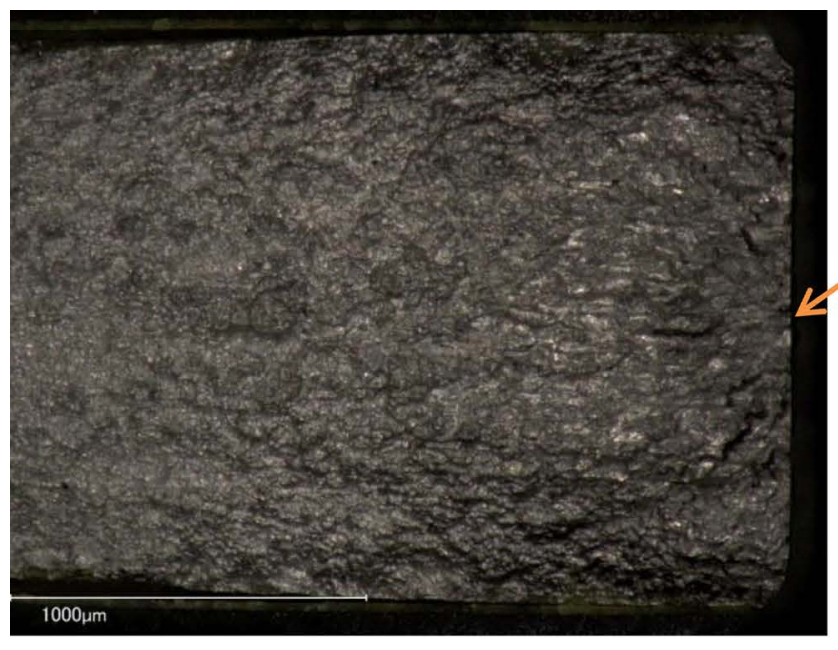

(a)

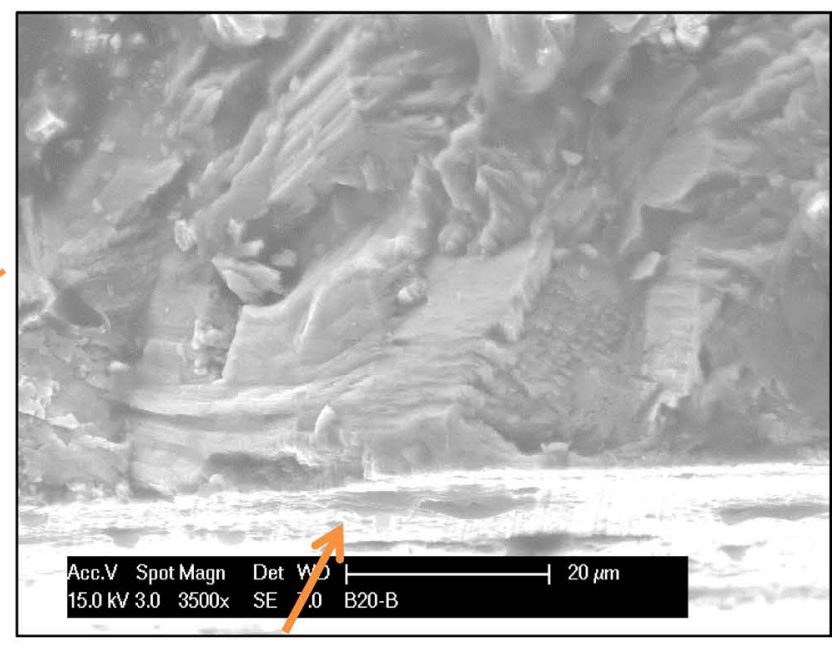

(b)

Figure 6. Fracture surface of the as-painted fatigue specimen B20. (a) As-painted (baseline). The arrow is pointing to the nucleation site at the edge (thickness of specimen); (b) Higher magnification of nucleation site under SEM, indicating a potential rough surface due to machining as source of fatigue crack nucleation (orange arrow at tilted image). 


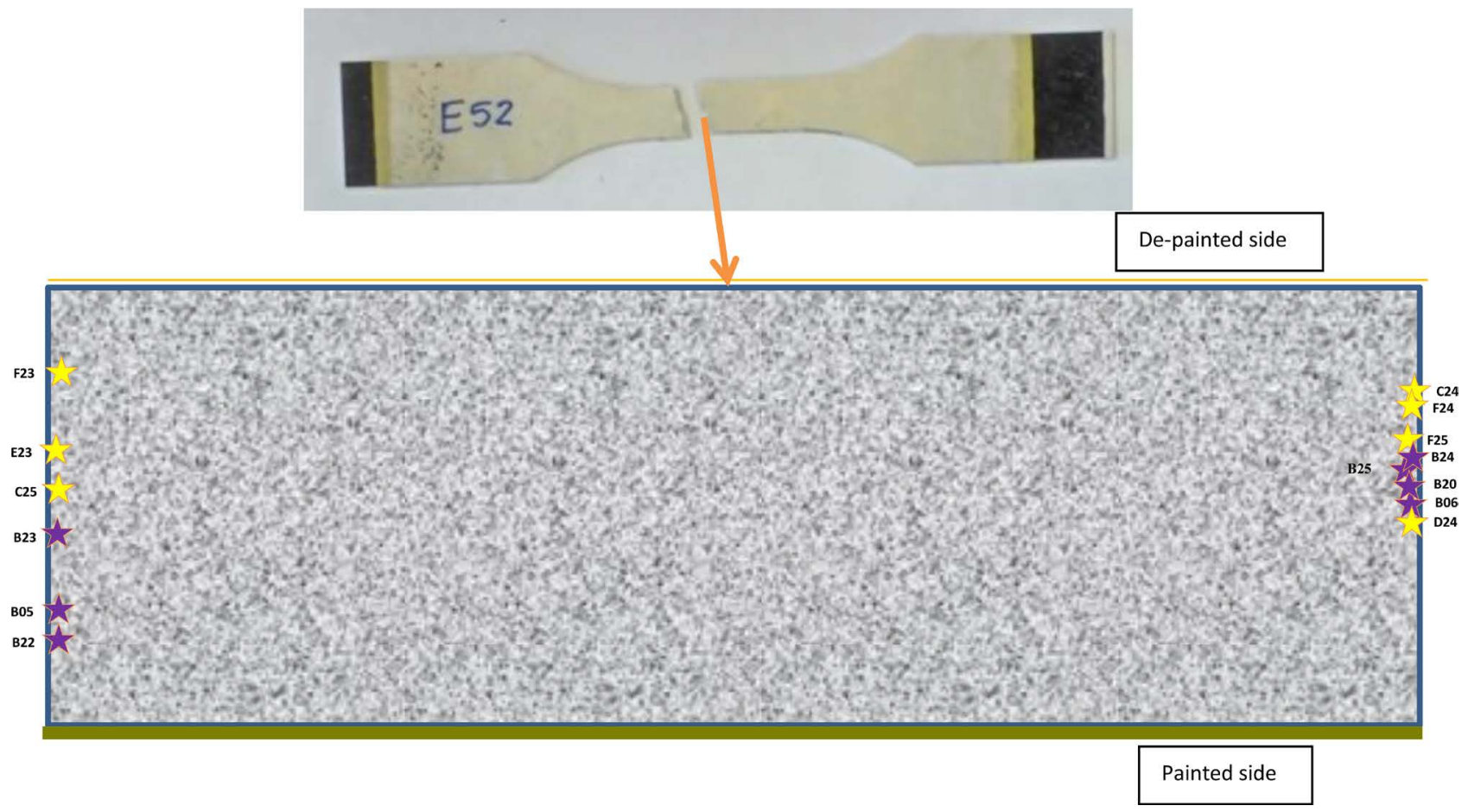

Figure 7. Schematic showing the fatigue nucleation sites for de-painted (yellow star) and painted (purple star) specimens. For de-painted specimens, the de-painted side is shown on top with thin orange line.

for both baselines painted and five cycle de-painted specimens were on the edges of the fatigue specimens. The fractography suggested that all the nucleation sites occurred at the machined edges, as the classic location of fatigue nucleation sites.

The failure origins for the baseline and AP treated specimens were particles or machining marks located along the side machined edge of the specimen, i.e. not on the painted/de-painted faces, as shown schematically in Figure 7. For de-painted specimens, the plasma de-painted face is shown on top. The yellow star designates the fatigue crack nucleation sites for de-painted specimens and purple stars indicate the crack origins for not-treated as-painted specimens. Additionally as evident in Figure 7, fatigue crack nucleation sites were found to occur equally at both edges. Therefore, it may be concluded that the de-painting process did not play a key role in the fatigue nucleation process and performance of the tested specimens.

\section{Concluding Remarks}

The objective of this study was to determine whether paint removal using Atmospheric Plasma was beneficial, detrimental, or neutral to the fatigue properties of aluminium substrates.

As the plasma paint removal process requires many passes over the same painted area, the substrate can become extremely hot, for minutes at a time, depending on the surface area and the material of the de-painted section. With high heat comes the danger of altering the heat treatment of the metal and consequently changing its microstructure. Conductivity measurements reported in 
[4] [5] [6] [7], indicated that the AP process parameters used in this study, would not have been sufficient to change the precipitation condition (temper) of the 2024-T3 aluminium alloy sheet material.

The atmospheric plasma processing parameters used were representative of a procedure for removing the top coat only while retaining the majoring of the primer intact. Since the test panels were not repainted between paint removal cycles, the paint removal was considered to be a more aggressive situation than had the panels been repainted between cycles. As a result, the five AP treatment cycles, which were conducted in succession, did not allow the test panels and test fixtures to cool until all five cycles were completed.

The fractography suggested that crack nucleation sites had typically originated at machined edges from discontinuities in the 2024-T3 aluminium alloy. Since all the nucleation sites occurred on the machined edges, it may be concluded that the de-painting process did not play a key role in the fatigue nucleation process and performance of the tested specimens.

Visual comparison of the Stress-Life curves determined according to ASTM E739 suggest that plasma de-painting process does not have a significant detrimental effect on the fatigue life. The results indicated no definitive relationship between AP de-painting and crack nucleation due to cyclic loading, and the process did not affect the location of nucleation sites in the aluminium alloy.

This limited paint removal study has determined that the AP process has the potential to replace current hazardous and less environmentally friendly paint removal methods; though a full systematic qualification and evaluation process is still required for it to be considered as an accepted industrial paint removal process. This conclusion is encouraging for the AP de-painting process and supports the technology's emergence into industrial applications for de-painting. For this to occur, the range of process conditions corresponding to the minimum and maximum severity of paint removal process has to be established for each particular combination of paint scheme and substrate.

\section{Acknowledgements}

We appreciate the efforts and assistance with fractography and microscopy of OCdt Daphne Servant from the Royal Military College. The fatigue testing and data gathering was performed by Dmitrii Klishch is also recognized and appreciated. The present work is performed as a contribution to the DND/DRDC funded projectAl-004516.

\section{References}

[1] SAE (2012) Paint Stripping of Commercial Aircraft-Evaluation of Materials and Processes.

[2] Merati, A., Yanishevsky, M., Despinic, T. and Lo, P. (2017) The Effect of Atmospheric Plasma Paint Stripping on the Fatigue Crack Growth Properties of Aluminium Substrates. Journal of Minerals and Materials Characterization and Engineering (JMMCE), 5. https://doi.org/10.4236/jmmce.2017.54014 
[3] Merati, A., Yanishevsky, M., Despinic, T., Lo, P. and Pankov, V. Alternate Environmentally Friendly De-Painting Process for Aircraft Structures-Atmospheric Plasma. Journal of Minerals and Materials Characterization and Engineering (JMMCE), In Press.

[4] Merati, A., Yanishevsky, M., Despinic, T. and Lo, P. (2016) Metallographic Analysis of Paint Stripping Techniques-Atmospheric Plasma.

[5] Merati, A., Lo, P., Despinic, T., Genest, M. and Yanishevsky, M. (2016) Effect of Paint Removal Technique on Crack Detectability-Liquid Penetrant Inspection.

[6] Merati, A., Despinic, T., Lo, P., Yanishevsky, M. and Genest, M. (2016) Effect of Atmospheric Plasma Paint Stripping on Fatigue Properties of Aluminium Substrates.

[7] Merati, A., Despinic, T., Lo, P., Pankov, V. and Yanishevsky, M. (2016) Preliminary Investigation for Effective Atmospheric Plasma De-painting Process Parameters.

[8] Merati, A., Despinic, T., Lo, P., Pankov, V. and Yanishevsky, M. (2016) Atmospheric Plasma-An Alternative Paint Stripping Process for Aircraft Structures. International Conference \& Exhibition on Advanced \& Nano Materials (ICANM2016) Proceedings, Montreal, August 2016, 61-72.

[9] Merati, A., Lo, P., Despinic, T. and Yanishevsky, M. (2016) Effect of Paint Removal Process on Crack Detectability Using Liquid Penetration Inspection Method. International Conference \& Exhibition on Advanced \& Nano Materials (ICANM2016) Proceedings, Montreal, August 2016, 48-59.

[10] American Society for Testing and Materials (2015) Standard Practice for Conducting Force Controlled Constant Amplitude Axial Fatigue Tests of Metallic Materials. STM International, West Conshohocken. www.astm.org

[11] Rice, R.C., Jackson, J.L., Bakuckas, J. and Thompson, S. (2003) Metallic Materials Properties Development and Standardization (MMPDS). Office of Aviation Research, Washington DC.

[12] American Society for Testing and Materials (2015) Standard Practice for Statistical Analysis of Linear or Linearized Stress-Life (S-N) and Strain-Life $(\varepsilon-\mathrm{N})$ Fatigue Data. ASTM International, West Conshohocken. www.astm.org

[13] Yanishevsky, M., Merati, A. and Bombardier, Y. (2017) Fatigue Testing of Baseline and Atmospheric Plasma Treated 2024-T3 Aluminium Alloy Sheet. 